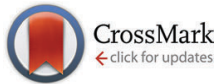

Cite this: Phys. Chem. Chem. Phys., 2016, 18, 5179

Received 30th December 2015 Accepted 15th January 2016

DOI: $10.1039 / \mathrm{c} 5 \mathrm{cp} 08041$

www.rsc.org/pccp

\section{Carrier separation and charge transport characteristics of reduced graphene oxide supported visible-light active photocatalysts $\dagger$}

\author{
Ramalingam Vinoth, ${ }^{a}$ Peramaiah Karthik, ${ }^{a}$ Chellamuthu Muthamizhchelvan, ${ }^{b}$ \\ Bernaurdshaw Neppolian ${ }^{\star a}$ and Muthupandian Ashokkumar*c
}

\begin{abstract}
Extending the absorption to the visible region by tuning the optical band-gap of semiconductors and preventing charge carrier recombination are important parameters to achieve a higher efficiency in the field of photocatalysis. The inclusion of reduced graphene oxide (rGO) support in photocatalysts is one of the key strategies to address the above-mentioned issues. In this study, rGO supported Agl-meso$\mathrm{TiO}_{2}$ photocatalysts were synthesized using a sonochemical approach. The physical effects of ultrasound not only improved the crystallinity of $\mathrm{Agl}-\mathrm{mesOTiO} \mathrm{T}_{2}$ but also increased the surface area and loading of the $\mathrm{Agl}-\mathrm{mesoTiO}_{2}$ nanocomposite on $\mathrm{rGO}$ sheets. The low intense oxygen functionalities $(\mathrm{C}-\mathrm{O}-\mathrm{C}$ and $\mathrm{COOH}$ groups) peak observed in the high resolution $\mathrm{Cls}$ spectrum of a hybrid $\mathrm{Agl}-\mathrm{mesOTiO}_{2}-\mathrm{rGO}$ photocatalyst clearly confirmed the successful reduction of graphene oxide (GO) to rGO. The interfacial charge transfer between the $\mathrm{rGO}$ and the $\mathrm{p}-\mathrm{n}$ junction of heterostructured photocatalysts has decreased the band-gap of the photocatalyst from 2.80 to $2.65 \mathrm{eV}$. Importantly, the integration of rGO into Agl-mesoTiO 2 composites serves as a carrier separation centre and provides further insight into the electron transfer pathways of heterostructured nanocomposites. The individual effects of photo-generated electrons and holes over rGO on the photocatalytic degradation efficiency of rhodamine (RhB) and methyl orange (MO) using Agl-mesoTiO $-\mathrm{rGO}$ photocatalysts were also studied. Our experimental results revealed that photo-generated superoxide $\left(\mathrm{O}_{2}^{-\bullet}\right)$ radicals are the main reactive species for the degradation of $\mathrm{MO}$, whereas photo-generated holes $\left(\mathrm{h}^{+}\right)$are responsible for the degradation of RhB. As a result, $60 \%$ enhancement in $\mathrm{MO}$ degradation was observed in the presence of $\mathrm{rGO}$ in comparison to that of the pure Agl-mesoTiO 2 photocatalyst. This is due to the good electron acceptor and the ultrafast electron transfer properties of $\mathrm{rGO}$ that can effectively reduce the molecular oxygen to produce a large amount of reactive $\mathrm{O}_{2}{ }^{-\bullet}$ radicals. However, in the case of $\mathrm{RhB}$ degradation, $\mathrm{h}^{+}$is the main reactive species which showed a slightly increased photocatalytic activity $(12 \%)$ in the presence of $\mathrm{rGO}$ support where the role of $\mathrm{rGO}$ is almost negligible. This study suggests the effective roles of $\mathrm{rGO}$ for the degradation of organics, i.e., the rate of photocatalytic degradation also depends on the nature of compound rather than rGO support.
\end{abstract}

\section{Introduction}

Methyl orange (MO) and rhodamine B (RhB) are commonly used dyes in textile industries, which are toxic and carcinogenic in nature. The expulsion of these dyes to the ground water

\footnotetext{
${ }^{a}$ SRM Research Institute, SRM University, Kattankulathur, Kancheepuram 603203 (D.t.), Tamil Nadu, India. E-mail: neppolian.b@res.srmuniv.ac.in; Fax: +91-44-2745-6702; Tel: +91-44-2741-7916

${ }^{b}$ Center for Materials Science and Nano Devices, Department of Physics, SRM University, Kattankulathur, Kancheepuram 603203 (D.t.), Tamil Nadu, India ${ }^{c}$ The School of Chemistry, University of Melbourne, Parkville, Melbourne, Victoria 3010, Australia. E-mail: masho@unimelb.edu.au; Fax: +61-9347-5180; Tel: $+61-8344-7090$

$\dagger$ Electronic supplementary information (ESI) available. See DOI: 10.1039/c5cp08041j
}

system by industries causes severe health issues. These textile dyes are very difficult to degrade because of their complex structures. ${ }^{1-3}$ The photocatalytic degradation of organic pollutants using semiconductor photocatalysts is one of the environmentally friendly techniques. ${ }^{4,5} \mathrm{TiO}_{2}$ is the most promising photocatalyst owing to its attractive properties such as good catalytic activity, strong oxidizing capability, high stability and non-toxic nature., $\mathrm{TiO}_{2}$ with different nanostructures has been used as effective photocatalysts. Kim et al. reported that mesoporous $\mathrm{TiO}_{2}$ with a high surface area showed better photocatalytic performance than commercial Degussa P25 $\left(\mathrm{TiO}_{2}\right)^{8}$

In recent years, low band gap silver-halide ( $\mathrm{AgI}, \mathrm{AgCl}$ and $\mathrm{AgBr}$ ) based composites have been used as visible light active photocatalysts. ${ }^{9-14}$ Among them, AgI has been considered as a 
promising visible light photocatalyst because of its smaller band gap than $\mathrm{AgBr}$ and $\mathrm{AgCl}{ }^{15}$ It has been intensively studied that $\beta$-AgI is a direct band-gap semiconductor, while $\mathrm{AgBr}$ and $\mathrm{AgCl}$ are indirect bandgap semiconductors. Hence, the absorption co-efficient of the direct bandgap semiconductor material is higher than indirect bandgap semiconductors. ${ }^{16}$ This suggests that direct bandgap materials are much more efficient for photocatalytic applications over indirect bandgap materials. The blending of AgI with $\mathrm{TiO}_{2}$ considerably shifts the absorption of $\mathrm{TiO}_{2}$ towards the visible region. ${ }^{17} \mathrm{Hu}$ et al. synthesized visible light active $\mathrm{AgI}-\mathrm{TiO}_{2}$ photocatalysts using the depositionprecipitation method for the degradation of azo dyes. ${ }^{18} \mathrm{Li}$ et al. prepared highly efficient nanostructured $\mathrm{AgI}-\mathrm{TiO}_{2}$ visible light active photocatalysts for the degradation of crystal-violet and 4-chlorophenol. ${ }^{19}$ The AgI-TiO ${ }_{2}$ composite was also prepared using the same deposition-precipitation method and it was found to be a stable photocatalyst with bactericidal properties under visible light irradiation. ${ }^{20}$ Moreover, AgI with different morphological structures has also been demonstrated as an effective visible light active photocatalyst. ${ }^{9,21}$

In order to further increase the photocatalytic activity of silver and silver halide based photocatalysts, graphene oxide (GO) has been used as a solid support. ${ }^{22}$ Graphene is a two dimensional material made up of carbon atoms with a honeycomb lattice structure. The two dimensional graphene sheets possess interesting electronic properties such as zero bandgap, high charge carrier mobility and zero effective mass. ${ }^{23-25}$ In addition to this, graphene could also dissociate the excited charge carrier at very fast rate to increase the photocatalytic activity. Recently, Wang et al. prepared the $\mathrm{Ag}-\mathrm{AgBr} / \mathrm{TiO}_{2} / \mathrm{rGO}$ nanocomposite using the solvothermal-photoreduction method and demonstrated it as an efficient photocatalyst for the degradation of penicillin $\mathrm{G}$ in the presence of visible light. ${ }^{26}$ Wang and co-workers synthesized visible light active $\mathrm{AgBr}$ coupled with graphene oxide sheets for the degradation of aqueous rhodamine B. $^{27}$ However, there is no report available for the preparation of graphene supported $\mathrm{AgI}-\mathrm{TiO}_{2}$ photocatalysts using ultrasound for the degradation of organic pollutants. As mentioned earlier, the use of AgI has several advantages over AgBr. The use of ultrasound during the preparation of metal-oxides could enhance the rate of hydrolysis and may lead to the formation of metal-oxide composites with improved crystalline phase, high surface area and controlled size distribution with unique morphologies, etc. ${ }^{28}$ Moreover, the shear forces produced by ultrasound helps in incorporating the metal and metal-oxide nanoparticles over rGO support. ${ }^{29}$ Hence, the current research work is mainly focused on the synthesis and photocatalytic properties of $\mathrm{AgI}-\mathrm{mesoTiO}_{2}-\mathrm{rGO}$ photocatalysts.

\section{Experimental section}

\subsection{Materials}

Graphite flakes (particle size +100 mesh $(\geq 75 \% \min )$ ) were purchased from Sigma Aldrich. Analytical grade silver nitrate and titanium tetraisopropoxide were purchased from SRL
Chemicals, India and analytical grade potassium iodide was obtained from Rankem, India. All other reagents and solvents were of analytical grade and were used as received without any further purification.

\subsection{Synthesis of graphene oxide (GO)}

GO was synthesized using modified Hummer's method. ${ }^{30}$ Briefly, $2 \mathrm{~g}$ of graphite powder and $1 \mathrm{~g}$ of $\mathrm{NaNO}_{3}$ were added to $46 \mathrm{~mL}$ of conc. $\mathrm{H}_{2} \mathrm{SO}_{4}$. This mixture was stirred for $30 \mathrm{~min}$. $6 \mathrm{~g}$ of $\mathrm{KMnO}_{4}$ was then added under ice cold conditions to prevent the temperature not exceed $20{ }^{\circ} \mathrm{C}$, after that this suspension was stirred at $35{ }^{\circ} \mathrm{C}$ for $30 \mathrm{~min}$. Furthermore, $92 \mathrm{~mL}$ of deionized water was slowly added and the reaction mixture was heated up to $98{ }^{\circ} \mathrm{C}$ for $30 \mathrm{~min}$. To remove excess $\mathrm{KMnO}_{4}$, an appropriate amount of $3 \%$ aqueous $\mathrm{H}_{2} \mathrm{O}_{2}$ solution was added dropwise into the mixture until no bubbles were observed. The mixture was centrifuged and washed with deionized water until neutral $\mathrm{pH}$ was reached. Finally, the collected product was freeze dried.

\subsection{Synthesis of mesoporous $\mathrm{TiO}_{2}$}

Mesoporous $\mathrm{TiO}_{2}$ was prepared as reported in a previous report. ${ }^{31}$ In brief, titanium tetraisopropoxide $(0.032 \mathrm{~mol})$ and glacial acetic acid $(0.016 \mathrm{~mol})$ were dissolved in $20 \mathrm{~mL}$ of absolute ethanol and the mixture was stirred for $1 \mathrm{~h}$. Then this mixture was added to $100 \mathrm{~mL}$ of deionized water drop by drop under sonication. During the whole process, the sonication cell was kept in an ice bath to avoid overheating. The mixture was sonicated for $3 \mathrm{~h}$ (3 s on, $1 \mathrm{~s}$ off, amplitude 40\%) using a $13 \mathrm{~mm}$ diameter high intensity probe (Sonics and Materials, VCX-750, $20 \mathrm{KHz}$ ). The products were collected by centrifugation and washed with Milli-Q water several times and dried at $100{ }^{\circ} \mathrm{C}$ using a hot air oven. Finally, the as prepared powder was calcined at $400{ }^{\circ} \mathrm{C}$ for $1 \mathrm{~h}$.

\subsection{Synthesis of AgI-mesoTiO ${ }_{2}$ and $\mathrm{AgI}-\mathrm{mesoTiO}_{2}-\mathrm{rGO}$ composites}

$103 \mathrm{mg}$ of $\mathrm{KI}$ and $500 \mathrm{mg}$ of as prepared mesoporous $\mathrm{TiO}_{2}$ were added to $50 \mathrm{~mL}$ of DI water under constant stirring. Then this mixture was stirred for $30 \mathrm{~min}$ and then sonicated for $30 \mathrm{~min}$ using an ultrasonic bath (Labman scientific, $40 \mathrm{kHz}, 250 \mathrm{~W}$ ). To this mixture, a required amount of GO and $5 \mathrm{~mL}$ of $22 \mathrm{mg} \mathrm{mL}^{-1}$ $\mathrm{AgNO}_{3}$ solution were added. The above mixture was sonicated for $1 \mathrm{~h}$, centrifuged, washed with Milli-Q water several times and dried at $80{ }^{\circ} \mathrm{C}$ for $12 \mathrm{~h}$. Similarly, the AgI-mesoTiO ${ }_{2}$ photocatalyst was prepared as reported earlier with a minor modification. ${ }^{32}$ Finally, the prepared AgI-mesoTiO ${ }_{2}$ and AgI-mesoTiO $2-$ rGO samples were calcinated at $350{ }^{\circ} \mathrm{C}$ for $2 \mathrm{~h}$. For comparison, the AgI-mesoTiO ${ }_{2}-\mathrm{rGO}$ photocatalyst was also prepared in the absence of ultrasound.

\subsection{Characterization}

Diffuse reflectance spectra (DRS) were recorded using a Shimadhzu UV-2600 UV-vis spectrophotometer in the DRS mode. X-ray diffraction (XRD) patterns were recorded on a PANaltical X'pert powder diffractometer using $\mathrm{Cu} \mathrm{K} \alpha$ radiation. The morphological 
studies with energy dispersive spectrum (EDX) were analyzed using a Field Emission-Scanning Electron Microscope (FEI Quanta FEG 200 HR-SEM). Transmission electron microscopic (TEM) images were taken using JEM-2100 JEOL, Japan. Raman spectra were obtained using (Horiba Jobin Yvon) and He-Ne laser $(\lambda=630 \mathrm{~nm})$. Fourier Transform Infrared (FTIR) analysis was carried out using a FTIR spectrophotometer (Agilent resolution pro). X-ray photoelectron spectroscopy (XPS) measurements were obtained using K-alpha instruments, USA, $\mathrm{Al}$ as a source, size 400 microns and range 0 to $1350 \mathrm{eV}$. The surface area and average pore size of the photocatalyst were measured using a Quantachrome Nova-1000 surface analyzer. The photocatalytic degradation of MO and RhB was monitored using a UV-visible spectrophotometer (Specord-200 plus, Analytikjena, Germany).

\subsection{Photocatalytic activity}

For photocatalytic degradation reaction, methyl orange (MO) and rhodamine (RhB) were chosen as model pollutants. $80 \mathrm{mg}$ of the prepared photocatalyst was added to $80 \mathrm{~mL}$ of aqueous solution containing the appropriate dye $\left(10 \mathrm{mg} \mathrm{L}^{-1}\right.$ for $\mathrm{MO}$ and $5 \mathrm{mg} \mathrm{L}^{-1}$ for RhB). Prior to reaction, the dye solution with catalysts was stirred in the dark for $30 \mathrm{~min}$ to attain adsorptiondesorption equilibrium. The light source used for degradation was a $150 \mathrm{~W}$ tungsten lamp $(\lambda>420 \mathrm{~nm})$ [Heber scientific, India]. Samples were collected at regular time interval during irradiation, centrifuged and the clear solutions were transferred to a $3 \mathrm{~mL}$ quartz cuvette for measuring absorbance.

\subsection{Photoelectrochemical studies}

Photoelectrochemical measurements were carried out using an electrochemical workstation (CHI608E) in a conventional three electrode configuration with $\mathrm{Pt}$-wire and $\mathrm{Ag} / \mathrm{AgCl}$ (in saturated $\mathrm{KCl})$ as counter and reference electrodes, respectively. A $250 \mathrm{~W}$ Xe arc lamp (OSRAM, Germany) was utilized as the light source. A $0.1 \mathrm{M} \mathrm{Na}_{2} \mathrm{SO}_{4}$ aqueous solution was used as the electrolyte. For the preparation of the working electrode, $50 \mathrm{mg}$ of the photocatalyst was ground with $150 \mu \mathrm{L}$ of PEG (MW 400) and $125 \mu \mathrm{L}$ of ethanol to make slurry. Then, the slurry was coated on a $2.5 \times 2.5 \mathrm{~cm}^{2}$ fluorine-doped tin oxide (FTO) glass substrate with an active area of about $1 \times 1 \mathrm{~cm}^{2}$ by the doctor-blade method using scotch tape as a spacer. Finally, the electrode was dried in an oven and annealed at $350{ }^{\circ} \mathrm{C}$ for $45 \mathrm{~min}$.

\section{Results and discussion}

The XRD data of the synthesized photocatalysts are shown in Fig. 1. The mesoTiO ${ }_{2}$ exhibits characteristic diffraction peaks at $2 \theta$ values of $25.8,38,39.5,48,55,62.6,69.7,75.7$ and $83^{\circ}$, which correspond to (101), (004), (112), (200), (211), (213), (220), (215) and (312) planes, respectively. This result confirms that meso$\mathrm{TiO}_{2}$ exists in all the composites in a pure anatase form (JCPDS Card \#: 89-4921). The AgI nanoparticles in the composite show the diffraction patterns at $22.6,24,43$ and $46.6^{\circ}$ corresponding to the (100), (002), (103) and (112) planes, which could be attributed to the $\beta$-AgI structure (JCPDS Card \#: 78-1614). ${ }^{19}$

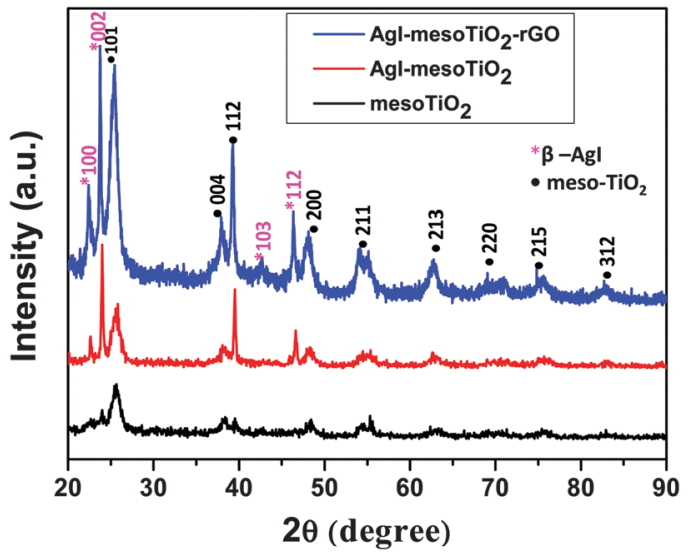

Fig. 1 XRD patterns of synthesized mesoTiO $2, \mathrm{Agl}-\mathrm{mesoTiO}_{2}$ and $\mathrm{Agl}-$ mesoTiO $2-r G O$ photocatalysts.

It is seen that $\beta$-AgI is the most prominent phase in both $\mathrm{AgI}-\mathrm{mesoTiO}_{2}$ and $\mathrm{AgI}-\mathrm{mesoTiO}{ }_{2}-\mathrm{rGO}$ composites. During calcination at $350{ }^{\circ} \mathrm{C}$, oxygen rich functional groups (epoxy groups) present on GO surfaces were reduced and $\mathrm{rGO}$ was obtained. ${ }^{33}$ However, no characteristic XRD peak for rGO was observed. This might be attributed to a less loading amount of rGO (4 wt $\%) \cdot{ }^{34-36}$ It is also very difficult to distinguish the diffraction pattern of rGO from the broader (101) plane of anatase $\mathrm{TiO}_{2} \cdot{ }^{37}$

Fig. 2(a) shows the UV-vis absorption spectra of the prepared photocatalysts recorded in DRS mode. The absorption edge of mesoTiO $_{2}$ is observed at $382 \mathrm{~nm}$, whereas both AgI-mesoTiO and $\mathrm{AgI}-\mathrm{mesOTiO}_{2}$-rGO composites exhibit a red shift and its absorption edge corresponds to 440 and $470 \mathrm{~nm}$, respectively. This shift in the absorption edge is due to the presence of AgI nanoparticles and rGO. Huang et al. reported that the chemical bonding between $\mathrm{TiO}_{2}$ and active sites of graphene causes a red shift in the absorption edge. ${ }^{35}$ Zhang et al. reported that the addition of graphene with $\mathrm{P}^{25}-\mathrm{TiO}_{2}$ shifted the absorption edge towards a higher wavelength region. ${ }^{38}$ As can be seen in Fig. 2(b), the calculated bandgap energies of mesoTiO $_{2}$, AgI-mesoTiO ${ }_{2}$ and AgI-mesoTiO ${ }_{2}-\mathrm{rGO}$ are 3.24, 2.80 and $2.65 \mathrm{eV}$, respectively. The slightly reduced bandgap of rGO loaded composite implies that unpaired $\pi$ electrons from graphene may bond with free electrons of $\mathrm{TiO}_{2}$. Meng et al. reported that graphene in $\mathrm{Ag}_{2} \mathrm{Se} / \mathrm{TiO}_{2}$ nanocomposites shifted the bandgap toward the visible region. ${ }^{39}$

The valence band (VB) and the conduction band (CB) potentials of semiconductors are two important factors for the effective separation of photogenerated electron-hole pairs to generate ${ }^{\bullet} \mathrm{OH}$ radicals and superoxide anions. The $\mathrm{VB}$ and $\mathrm{CB}$ potential edges were calculated using the following empirical formulae. ${ }^{40,41}$

$$
\begin{gathered}
E_{\mathrm{VB}}=\chi-E^{\mathrm{e}}+0.5\left(E_{\mathrm{g}}\right) \\
E_{\mathrm{CB}}=E_{\mathrm{VB}}-E_{\mathrm{g}}
\end{gathered}
$$

where $E_{\mathrm{VB}}$ and $E_{\mathrm{CB}}$ are the valence and conduction band edge potentials of a semiconductor, respectively, $\chi$ is the 

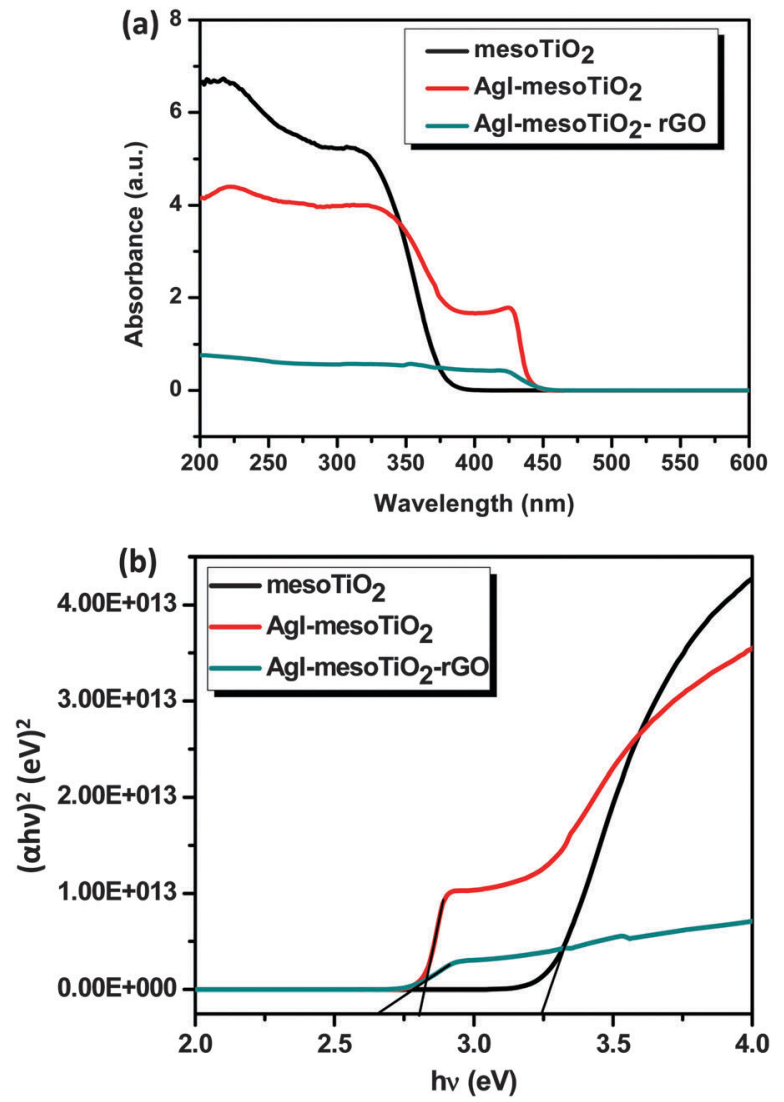

Fig. 2 (a) UV-vis absorption spectra (DRS mode) of mesoTiO ${ }_{2}$ AglmesoTiO ${ }_{2}$ and $\mathrm{Agl}-$ mesoTiO $2-\mathrm{rGO}$ photocatalysts and (b) plots between $(\alpha h \nu)^{2}(\mathrm{eV})^{2} v s . h \nu(\mathrm{eV})$ for the bandgap energy of mesoTiO $2, \mathrm{Agl}-\mathrm{mesoTiO}_{2}$ and $\mathrm{Agl}-\mathrm{mesOTiO}_{2}-\mathrm{rGO}$

electronegativity value of the semiconductor, which is the geometric mean of the electronegativities of constituent atoms, $E^{\mathrm{e}}$ is the energy of free electrons on the hydrogen scale $(\sim 4.5$ $\mathrm{eV}), E_{\mathrm{g}}$ is the band gap energy of the semiconductor. The calculated VB potentials of $\mathrm{TiO}_{2}$ and $\mathrm{AgI}$ are 2.95 and $2.38 \mathrm{eV}$. The CB potential of $\mathrm{TiO}_{2}(-0.29 \mathrm{eV})$ is lower than the $\mathrm{CB}$ of AgI $(-0.42 \mathrm{eV})$. Hence, the photogenerated electrons in the AgI can easily move to $\mathrm{TiO}_{2}$ through interfaces. Furthermore, the introduction of graphene in composites can effectively suppress the carrier recombination and thereby enhance the photocatalytic activity.

FE-SEM micrographs of mesoTiO ${ }_{2}, \mathrm{AgI}-\mathrm{mesoTiO}_{2}$ and AgImesoTiO ${ }_{2}-\mathrm{rGO}$ photocatalysts are shown in Fig. 3(a-d). As shown in Fig. 3(b) and (c) the small spherical shaped AgI nanoparticles are well anchored as well as uniformly distributed on the surface of $\mathrm{TiO}_{2}$ nanoparticles. It can be clearly seen from Fig. 3(c), rGO supported AgI-mesoTiO $\mathrm{O}_{2}$ prepared in the presence of ultrasound shows the formation of controllable and uniform thickness of the nanoparticles. ${ }^{42}$ However, the same nanocomposites prepared in the absence of ultrasound shows irregularshaped nanoparticle formation as displayed in Fig. 3(d). As shown in Fig. 3(e), the elemental X-ray analysis suggests that the rGO loaded composite composed of $\mathrm{Ti}, \mathrm{Ag}, \mathrm{I}, \mathrm{C}$ and $\mathrm{O}$.

The TEM images of the prepared $\mathrm{AgI}-\mathrm{mesoTiO}_{2}-\mathrm{rGO}$ are shown in Fig. 4(a) and (b). It can be clearly seen from Fig. 4(a) that the prepared nanocomposites are uniformly distributed on the $\mathrm{rGO}$ sheet. The average crystalline size of the AgI nanoparticle and mesoTiO $_{2}$ is 11 and $31 \mathrm{~nm}$. The magnified view of the AgI-mesoTiO ${ }_{2}-\mathrm{rGO}$ sample is given in Fig. 4(b). The interplanar spacing values of $\mathrm{TiO}_{2}$ and $\mathrm{AgI}$ nanoparticles are $0.344 \mathrm{~nm}$ and $0.351 \mathrm{~nm}$, which correspond to the (101) plane of the anatase $\mathrm{TiO}_{2}$ and $\beta$-AgI structure. ${ }^{19}$ This result is consistent with the XRD data. The TEM EDX [Fig. 4(c)] spectrum reconfirms that the AgI-mesoTiO $2-r G O$ composite is composed of $\mathrm{Ti}$, $\mathrm{Ag}, \mathrm{I}, \mathrm{C}$ and $\mathrm{O}$.

The FTIR spectra of GO, mesoTiO ${ }_{2}, \mathrm{AgI}-\mathrm{mesoTiO}_{2}$ and AgImesoTiO ${ }_{2}-\mathrm{rGO}$ are shown in Fig. 5(a). The FTIR spectrum of GO exhibits a broad absorption band located at $\sim 3440 \mathrm{~cm}^{-1}$, which corresponds to the stretching vibration of hydroxyl groups. The absorption peaks observed at 1631 and $1384 \mathrm{~cm}^{-1}$ are associated with the stretching vibration of aromatic $\mathrm{C}=\mathrm{C}$ and the bending vibration of $\mathrm{O}-\mathrm{H}$ groups, respectively. ${ }^{43}$ The absorption peaks centered at 1733, 1054 and $1118 \mathrm{~cm}^{-1}$ are related to the stretching vibrations of $\mathrm{C}-\mathrm{O}, \mathrm{C}-\mathrm{OH}$ and $\mathrm{C}=\mathrm{O}$ groups, respectively. ${ }^{44}$ The broad absorption band observed at around $350-750 \mathrm{~cm}^{-1}$ for all the photocatalysts except GO corresponds to the characteristic vibrations of $\mathrm{TiO}_{2}$. In addition, a broad band at 3441 and a sharp peak at $1624 \mathrm{~cm}^{-1}$ are assigned to the vibrations of surface adsorbed water moieties and hydroxyl groups present over $\mathrm{TiO}_{2} \cdot{ }^{31}$ The FTIR spectra of AgI-mesoTiO ${ }_{2}$ and AgI-mesoTiO ${ }_{2}-\mathrm{rGO}$ photocatalysts show an absorption band at $390-560 \mathrm{~cm}^{-1}$ which is assigned to the $\mathrm{Ag}-\mathrm{I}$ bond. More interestingly, the AgI-mesoTiO $-\mathrm{rGO}$ photocatalyst exhibits only the characteristic peaks of $\mathrm{TiO}_{2}$ and $\mathrm{AgI}$, whereas the intensities of the oxygen containing groups $(\mathrm{O}-\mathrm{H}, \mathrm{C}-\mathrm{O}$, $\mathrm{C}-\mathrm{OH}$ and $\mathrm{C}=\mathrm{O}$ ) have completely disappeared. These observations confirmed the successful reduction of GO to rGO.

The Raman spectra of GO, mesoTiO, AgI-mesoTiO ${ }_{2}$ and AgI-mesoTiO ${ }_{2}-\mathrm{rGO}$ are shown in Fig. 5(b). The mesoTiO exhibits five characteristic Raman bands at 144, 193, 398, 518 and $638 \mathrm{~cm}^{-1}$, which are mainly attributed to the anatase phase $\mathrm{TiO}_{2}$ structure. ${ }^{27}$ The intensities of the Raman bands of $\mathrm{TiO}_{2}$ are greatly suppressed in both AgI-mesoTiO ${ }_{2}$ and AgI-meso$\mathrm{TiO}_{2}$-rGO composites, which might be due to the loading of AgI with $\mathrm{TiO}_{2}$. GO shows the characteristic D and G bands at 1352 and $1591 \mathrm{~cm}^{-1}$, respectively. The $I_{\mathrm{D}} / I_{\mathrm{G}}$ value of GO was calculated to be 0.93. As shown in the inset of Fig. 5(b), D and G bands of rGO in AgI-mesoTiO ${ }_{2}$ are observed at 1352 and $1598 \mathrm{~cm}^{-1}$ and the corresponding $I_{\mathrm{D}} / I_{\mathrm{G}}$ value was estimated to be 0.97 . Therefore, the increase in the $I_{\mathrm{D}} / I_{\mathrm{G}}$ value of rGO supported AgI-mesoTiO ${ }_{2}$ compared to its $I_{\mathrm{D}} / I_{\mathrm{G}}$ value of GO clearly indicates that the incorporation of $\mathrm{TiO}_{2}$ and $\mathrm{AgI}$ induces defective sites on the surface of the rGO sheets.

XPS analysis was carried out to identify the chemical oxidation states of the elements ( $\mathrm{Ag}, \mathrm{I}, \mathrm{Ti}, \mathrm{C}$ and $\mathrm{O}$ ) and also to confirm the reduction of GO to rGO. As shown in Fig. 6(a), the XPS survey scan spectrum clearly indicates the existence of C, $\mathrm{O}, \mathrm{Ti}, \mathrm{Ag}$ and I elements in AgI-mesoTiO ${ }_{2}-\mathrm{rGO}$ nanocomposites. The Gaussian-Lorentz fitting was adopted to identify the constituents of the spectrum. Fig. 6(b) displays the high resolution C1s spectrum deconvoluted into five peaks. An intense 

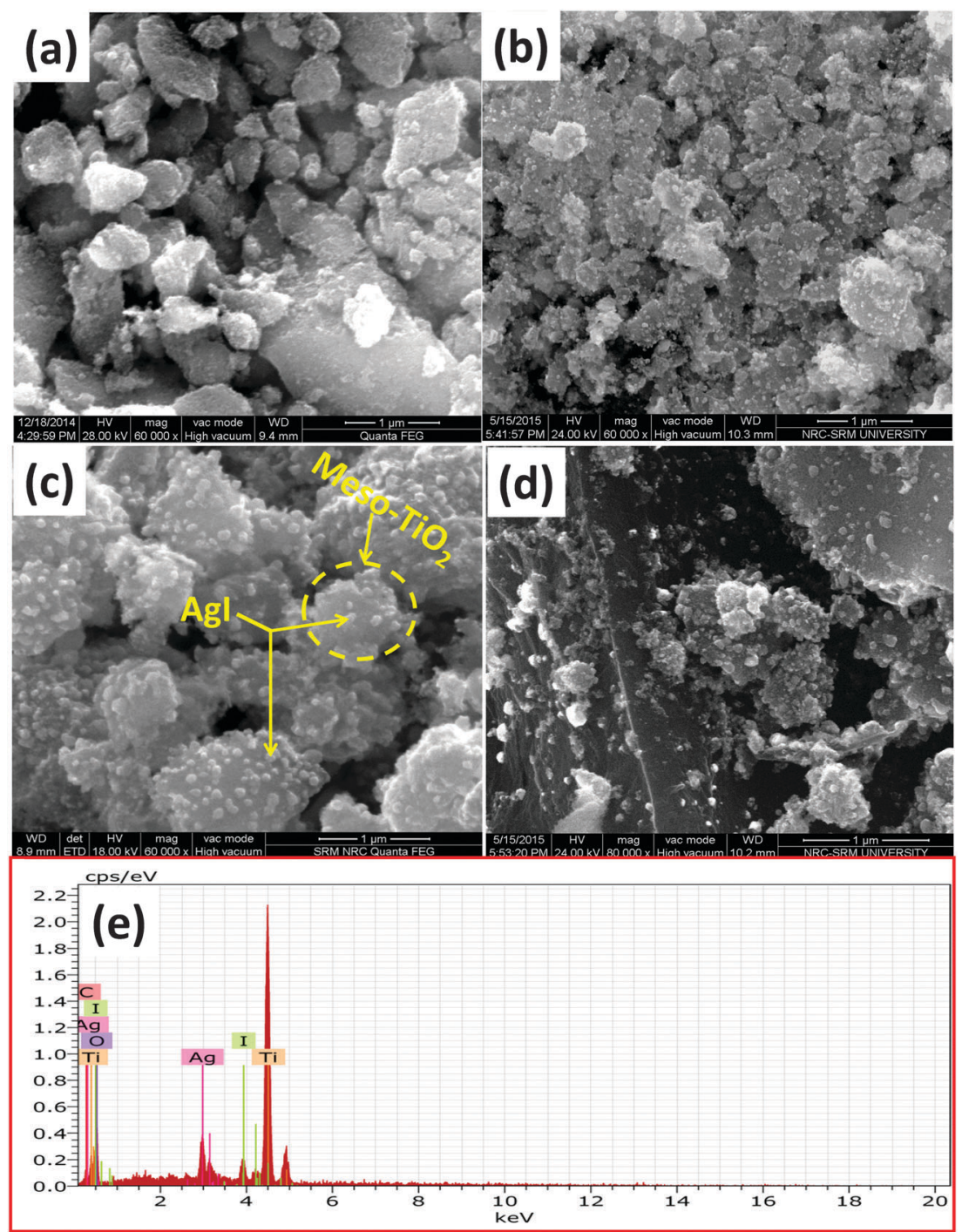

Fig. 3 FE-SEM images of (a) mesoTiO $_{2}$, (b) Agl-mesoTiO ${ }_{2}$, (c) Agl-mesoTiO $2-r G O$ prepared in the presence of ultrasound, (d) Agl-mesoTiO $2-r G O$ prepared in the absence of ultrasound and (e) EDX spectra of Agl-mesoTiO ${ }_{2}-\mathrm{rGO}$.

peak observed at $285.1 \mathrm{eV}$ corresponds to $\mathrm{C}-\mathrm{C}$ bonds. ${ }^{45}$ A low binding energy peak located at $282.2 \mathrm{eV}$ is attributed to the Ti-C bond. ${ }^{46}$ In addition, three more less intense binding energy peaks are noted at 283.5, 286.9 and $289.9 \mathrm{eV}$, which are assigned to $\mathrm{C}=\mathrm{C}, \mathrm{C}-\mathrm{O}-\mathrm{C}$ and $\mathrm{COOH}$ bonds, respectively. ${ }^{45}$ These results support the fact that the preparation of photocatalysts using the ultrasonication method followed by calcination considerably reduces the oxygen rich functional groups and thereby reduces GO into rGO. ${ }^{47} \mathrm{~A}$ similar result was also obtained in our previous studies on $\mathrm{CuO}-\mathrm{TiO}_{2}-\mathrm{rGO}$ and $\mathrm{Pt}$ loaded $\mathrm{TiO}_{2}-\mathrm{rGO}$ photocatalysts. ${ }^{29,47,48}$ Fig. 6(c) presents the high resolution O1s spectrum deconvoluted into four peaks with the binding energies centered at 528.7, 530.2, 530.9 and $531.8 \mathrm{eV}$, which are attributed to $\mathrm{O}=\mathrm{C}-\mathrm{OH}, \mathrm{Ti}-\mathrm{O}-\mathrm{Ti}, \mathrm{Ti}-\mathrm{O}-\mathrm{C}=\mathrm{O}$ and $\mathrm{Ti}-\mathrm{OH}$, respectively. ${ }^{47}$ As illustrated in Fig. 6(d), a high resolution $\mathrm{Ti} 2 \mathrm{p}$ spectrum exhibits two main peaks centered at 459.5 and $465.3 \mathrm{eV}$ corresponding to $\mathrm{Ti} 2 \mathrm{p}_{3 / 2}$ and $\mathrm{Ti} 2 \mathrm{p}_{1 / 2}$, respectively. The binding energy difference between the Ti $2 \mathrm{p}_{3 / 2}$ and
Ti $2 \mathrm{p}_{1 / 2}$ core level is calculated to be $5.7 \mathrm{eV}$, which clearly suggesting the presence of normal $\mathrm{Ti}^{4+}$ in anatase $\mathrm{TiO}_{2} \cdot{ }^{49,50}$ It is worth noting that these results are in good agreement with the XRD data, as shown in Fig. 1. Likewise, the high resolution spectrum of $\mathrm{Ag} 3 \mathrm{~d}$ exhibits two peaks at 369 and $375 \mathrm{eV}$ for $\mathrm{Ag}$ $3 \mathrm{~d}_{5 / 2}$ and $\mathrm{Ag} 3 \mathrm{~d}_{3 / 2}$, respectively [Fig. 6(e)]. These results clearly confirmed the presence of $\mathrm{Ag}^{+}$in $\mathrm{AgI}$, however there is no binding energy peak for metallic Ag observed. ${ }^{51}$ Similarly the high resolution I 3d spectrum shows the appearance of two peaks at 619.9 and $631.3 \mathrm{eV}$ which are assigned to $\mathrm{I}_{3 \mathrm{~d}} \mathrm{~d}$ and I $3 \mathrm{~d}_{3 / 2}$ [Fig. 6(f)], which confirms the $\mathrm{I}^{-}$state of iodine in AgI. ${ }^{19}$

The specific surface area, the average pore size and the total pore volume of the $\mathrm{AgI}-\mathrm{mesoTiO}_{2}-\mathrm{rGO}$ photocatalyst were analyzed through nitrogen adsorption-desorption isotherms. As shown in Fig. 7, the AgI-mesoTiO ${ }_{2}-\mathrm{rGO}$ composite exhibits a typical type (IV) isotherm pattern with an $\mathrm{H} 3$ hysteresis loop ranging from 0.44 to $0.88 P / P_{0}$. It is well known that the type (IV) isotherm is the characteristic isotherm of 

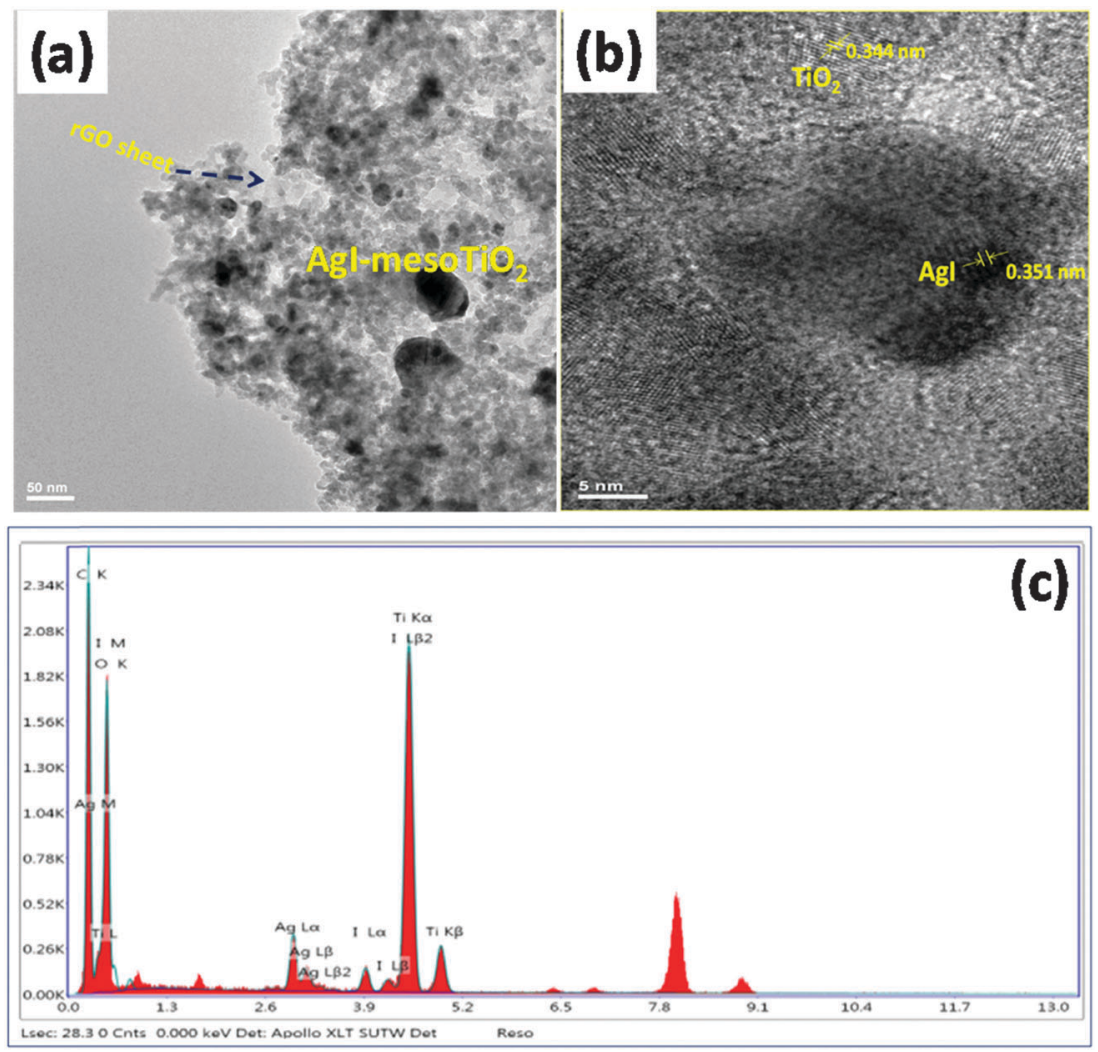

Fig. 4 (a) TEM images of synthesized $\mathrm{Agl}-$ mesoTiO $_{2}-\mathrm{rGO}$, (b) magnified view image of $\mathrm{Agl}-\mathrm{mesoTiO}_{2}-\mathrm{rGO}$ and (c) EDX of the Agl-mesoTiO $2-r G O$ photocatalyst.
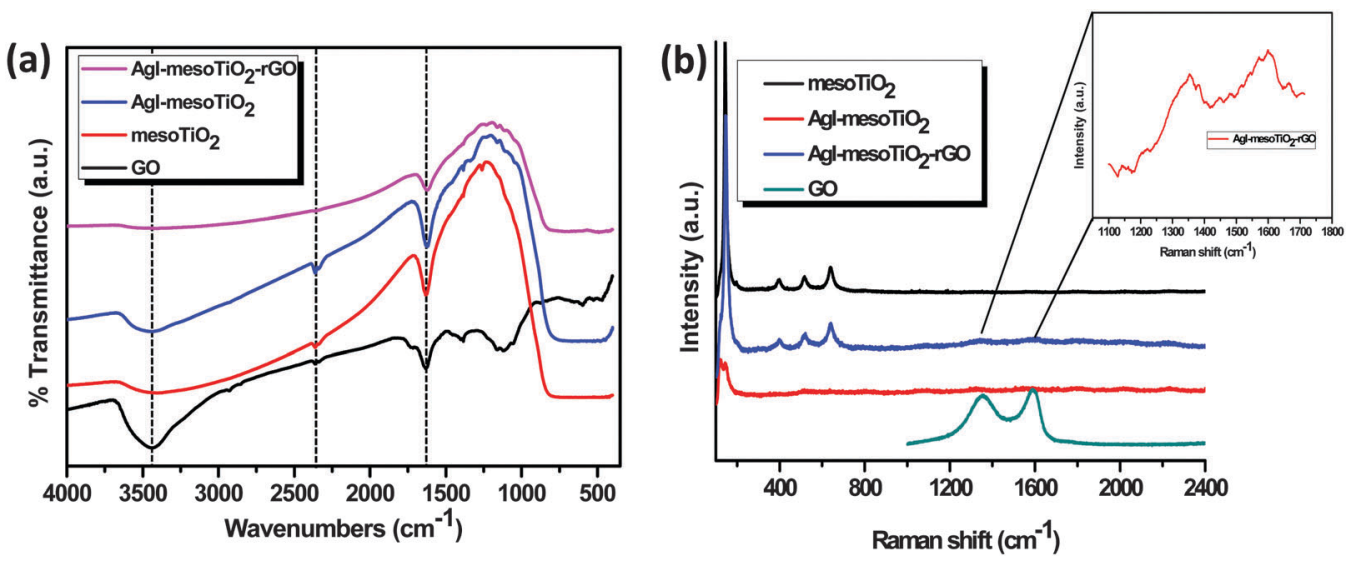

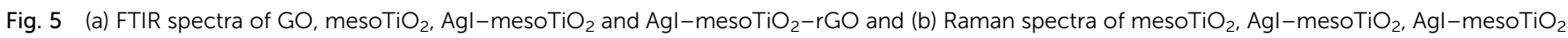
and GO. Inset (b) Raman spectra of $\mathrm{Agl}-\mathrm{mesOTiO}_{2}-\mathrm{rGO}$ showing the $1200-1700 \mathrm{~cm}^{-1}$ region.

mesoporous materials. ${ }^{52,53}$ The measured Brunauer-Emmett-Teller (BET) surface area of $\mathrm{AgI}-\mathrm{mesoTiO}_{2}-\mathrm{rGO}$ is $48.69 \mathrm{~m}^{2} \mathrm{~g}^{-1}$. The average pore size and the total pore volume of $\mathrm{AgI}-\mathrm{mesoTiO}_{2}-\mathrm{rGO}$ photocatalyst were calculated using the Barrett-Joyner-Halenda (BJH) method and the values are $8.8 \mathrm{~nm}$ and $0.108 \mathrm{cc} \mathrm{g}^{-1}$, respectively (inset Fig. 7). Thus, the mesoporous nature of the AgImesoTiO $_{2}-\mathrm{rGO}$ hybrid is clearly seen from the above observations.

The photocatalytic performance of the prepared catalysts was evaluated using an aqueous MO dye as a model compound under visible light irradiation. It is well known that the adsorption of dye molecules on the catalyst surface is one of the key factors for the degradation of organic pollutants. In particular, carbon based materials have shown excellent adsorption capacity. ${ }^{54}$ Compared to AgI-meso $\mathrm{TiO}_{2}$, the rGO loaded composite shows a higher adsorption of dye molecules (Fig. 8). This increase in dye adsorption on catalytic surfaces plays a significant role in achieving higher photocatalytic activity. Fig. 8(a) and (b) show a decrease in absorbance at $464 \mathrm{~nm}$ with an 

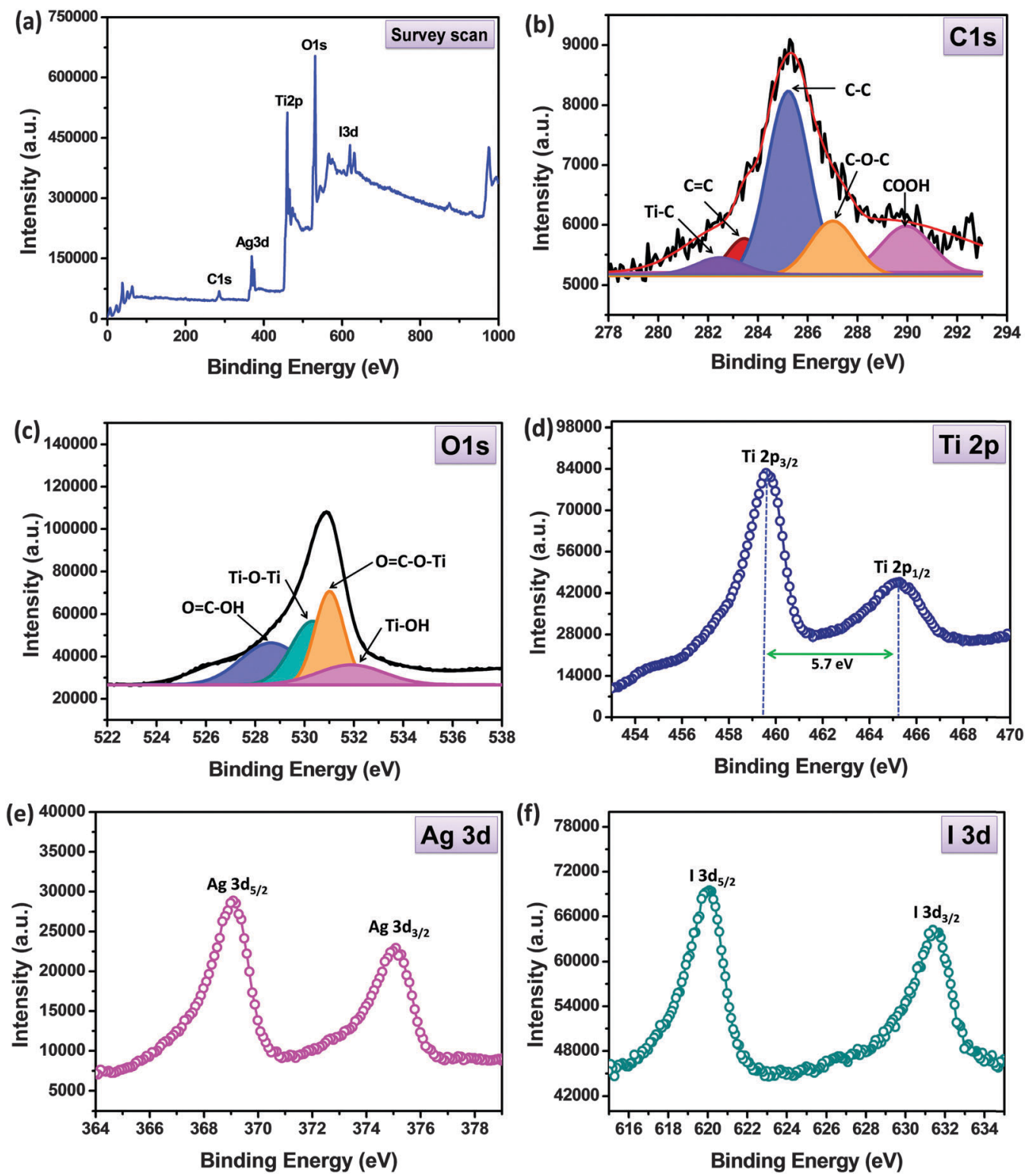

Fig. 6 XPS spectra of Agl-mesoTiO $2-r G O$ (a) survey scan, (b) high resolution C1s spectrum, (c) high resolution O1s spectrum, (d) high resolution Ti 2p spectrum, (e) high resolution $\mathrm{Ag} 3 \mathrm{~d}$ spectrum and (f) high resolution I 3d spectrum.

increase in irradiation time for AgI-mesoTiO 2 and $\mathrm{AgI}-\mathrm{meso}-$ $\mathrm{TiO}_{2}-\mathrm{rGO}$ composites under visible light irradiation. It can be observed that the photocatalytic degradation efficiency of AgImesoTiO 2 -rGO is substantially high.

As shown in Fig. 8(c), when experiments were carried out without a catalyst, no degradation of MO was observed indicating that MO was not degraded when exposed to visible light only as previously reported. ${ }^{55}$ This observation clearly attributes that the degradation of MO is only due to the action of the photocatalyst. In order to evaluate the effect of rGO loading, different weight percentages of $\mathrm{rGO}(0,1,2,4$ and $6 \mathrm{wt} \%)$ loaded AgI-mesoTiO 2 photocatalysts were prepared and their photocatalytic performance was also tested under identical experimental conditions. The composites with 1, 2 and $4 \mathrm{wt} \%$ rGO showed increased photocatalytic degradation compared to pure
AgI-mesoTiO 2 and mesoTiO ${ }_{2}$ photocatalysts, whereas $6 \mathrm{wt} \%$ rGO loaded AgI-mesoTiO ${ }_{2}$ photocatalysts decreased the photocatalytic activity. The reason is that a higher loading of rGO could increase the recombination rate instead of effective carrier separation thereby affecting the photocatalytic performance and also it masks the penetration of light into the particle. It can be seen in Fig. 8(c) that the $\mathrm{AgI}^{-}$mesoTiO $_{2}$ photocatalyst with $4 \mathrm{wt} \%$ rGO exhibits $92 \%$ MO degradation within 90 min but only 35\% of degradation occurred by AgImesoTiO ${ }_{2}$ under visible light irradiation. This $\sim 3$ fold increase in degradation was achieved due to the contribution of rGO. This is due to an efficient overlap of the conduction bands of $\mathrm{TiO}_{2}$ and rGO resulting in an effective separation of charge carriers between the graphene and the semiconductor $\left(\mathrm{TiO}_{2}\right)$ interface. Since $4 \mathrm{wt} \%$ rGO loaded $\mathrm{AgI}-\mathrm{mesoTiO}_{2}$ was found to 


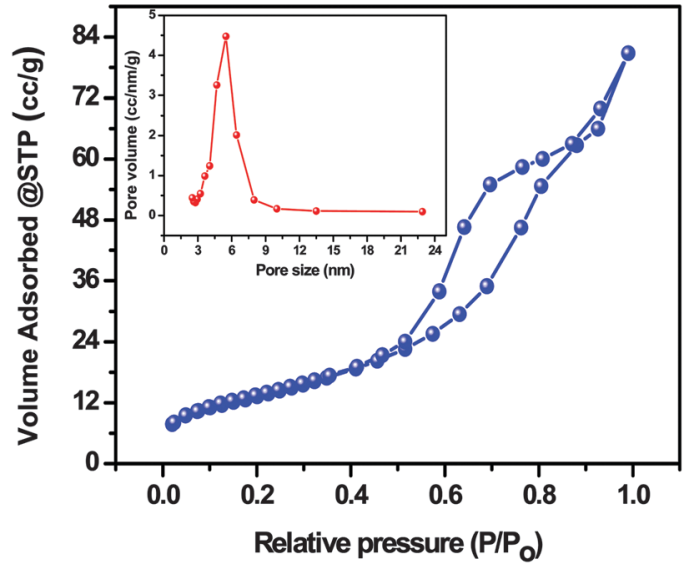

Fig. 7 Nitrogen adsorption-desorption isotherms of the $\mathrm{Agl}-\mathrm{mesoTiO}_{2}-$ $\mathrm{rGO}$ composite and the inset figure shows the pore size distribution and the pore volume.

show an optimum efficiency, rest of the studies were carried out with $4 \mathrm{wt} \%$ rGO loaded AgI-mesoTiO ${ }_{2}$ photocatalysts.

The synthesized mesoTiO 2 , AgI-mesoTiO ${ }_{2}$ and AgI-meso$\mathrm{TiO}_{2}-\mathrm{rGO}(4 \mathrm{wt} \%)$ photocatalysts were also demonstrated for $\mathrm{RhB}$ degradation. The prominent absorption peak centered at $554 \mathrm{~nm}$ gradually decreased with increasing irradiation time for AgI-mesoTiO ${ }_{2}$ and $\mathrm{AgI}-\mathrm{mesoTiO}_{2}-\mathrm{rGO}(4 \mathrm{wt} \%)$ photocatalysts
[Fig. 9(a) and (b)]. As shown in Fig. 9(c), the RhB with a catalyst - without light and with light - without a catalyst was also examined to investigate the role of the catalyst. No degradation of $\mathrm{RhB}$ could be observed either under visible light irradiation or alone. This suggests that the degradation reaction was effective due to photocatalysis. The mesoTiO ${ }_{2}$ does not exhibit photodegradation under visible light due to its wide bang gap which lies in the UV region. The AgI-mesoTiO ${ }_{2}$ gives only $\sim 88 \%$ of degradation within $10 \mathrm{~min}$. The $4 \mathrm{wt} \% \mathrm{rGO}$ loaded composite shows complete degradation ( $\sim 97 \%)$ of $\mathrm{RhB}$ in $10 \mathrm{~min}$. The presence of rGO in composites completely degraded the RhB within a very short span of time.

In general, during photocatalysis, hydroxyl radicals $\left({ }^{\circ} \mathrm{OH}\right)$ and superoxide anions $\left(\mathrm{O}_{2}^{-\bullet}\right)$ are the reactive species for the degradation of organic pollutants. In order to understand the role of $\mathrm{rGO}$ in the photocatalytic activity for the degradation of $\mathrm{RhB}$ and $\mathrm{MO}$ on the $\mathrm{AgI}-\mathrm{mesoTiO}_{2}-\mathrm{rGO}$ photocatalyst, a series of free radical trapping experiments were carried out, as shown in Fig. 10. In the presence of an ${ }^{\circ} \mathrm{OH}$ radical scavenger such as benzoic acid $(0.5 \mathrm{mM})$, only 12 and $20 \%$ degradation was decreased than that of the scavenger-free photocatalytic systems for RhB and MO, as shown in Fig. 10(a) and (b). These results clearly suggested that the photocatalytic degradation of both RhB and MO dyes was not only mediated through the ${ }^{\bullet} \mathrm{OH}$ radical reaction. In order to further examine the exact reactive
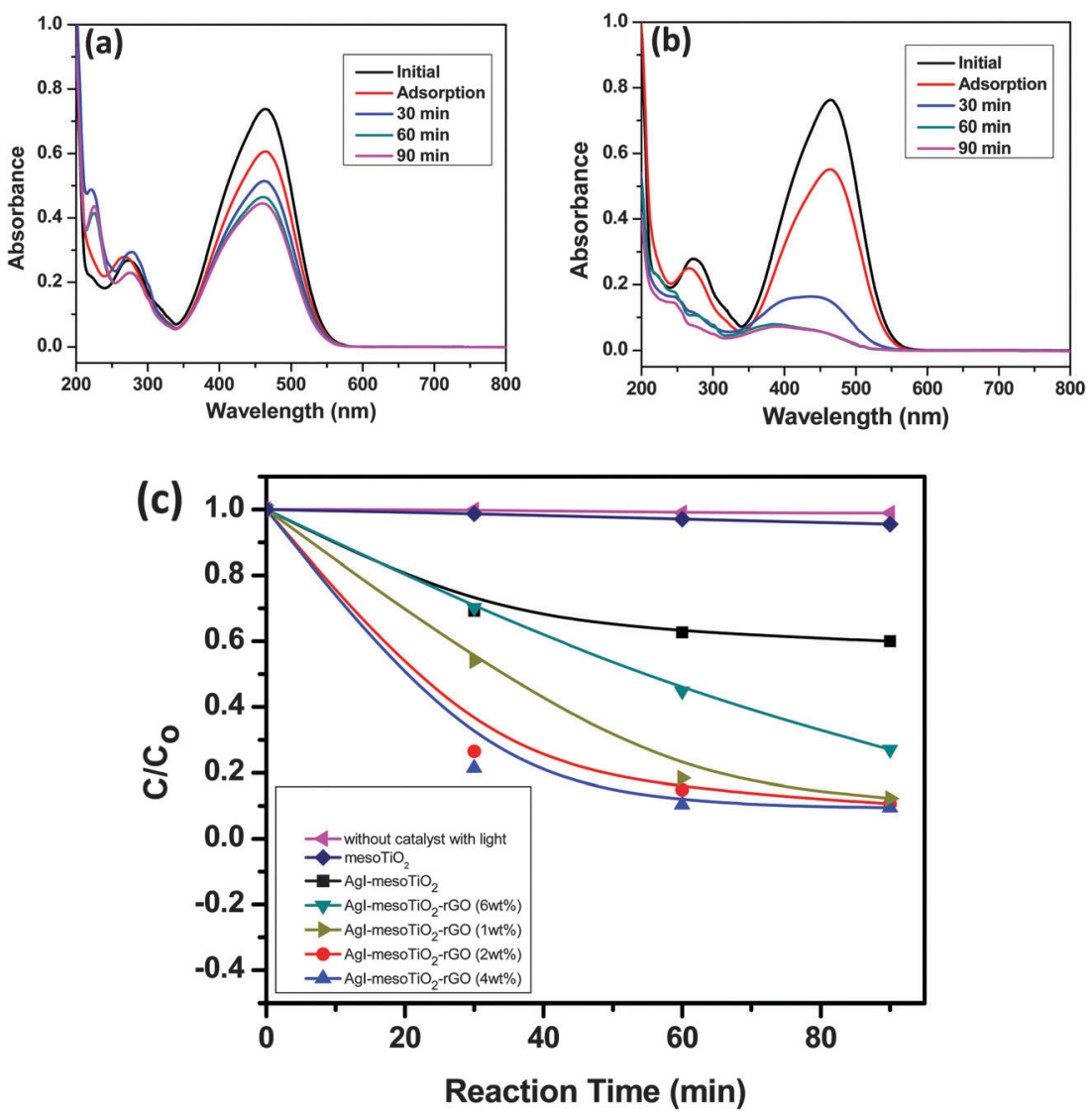

Fig. 8 Absorbance spectra of $\mathrm{MO}$ after photodegradation using (a) $\mathrm{Agl}-\mathrm{mesOTiO}_{2}$, (b) $\mathrm{Agl}-\mathrm{mesoTiO}_{2}-\mathrm{rGO}$ (4 wt\%) and (c) the degradation curve of the $\mathrm{MO}$ aqueous dye with different composites under visible light irradiation. 

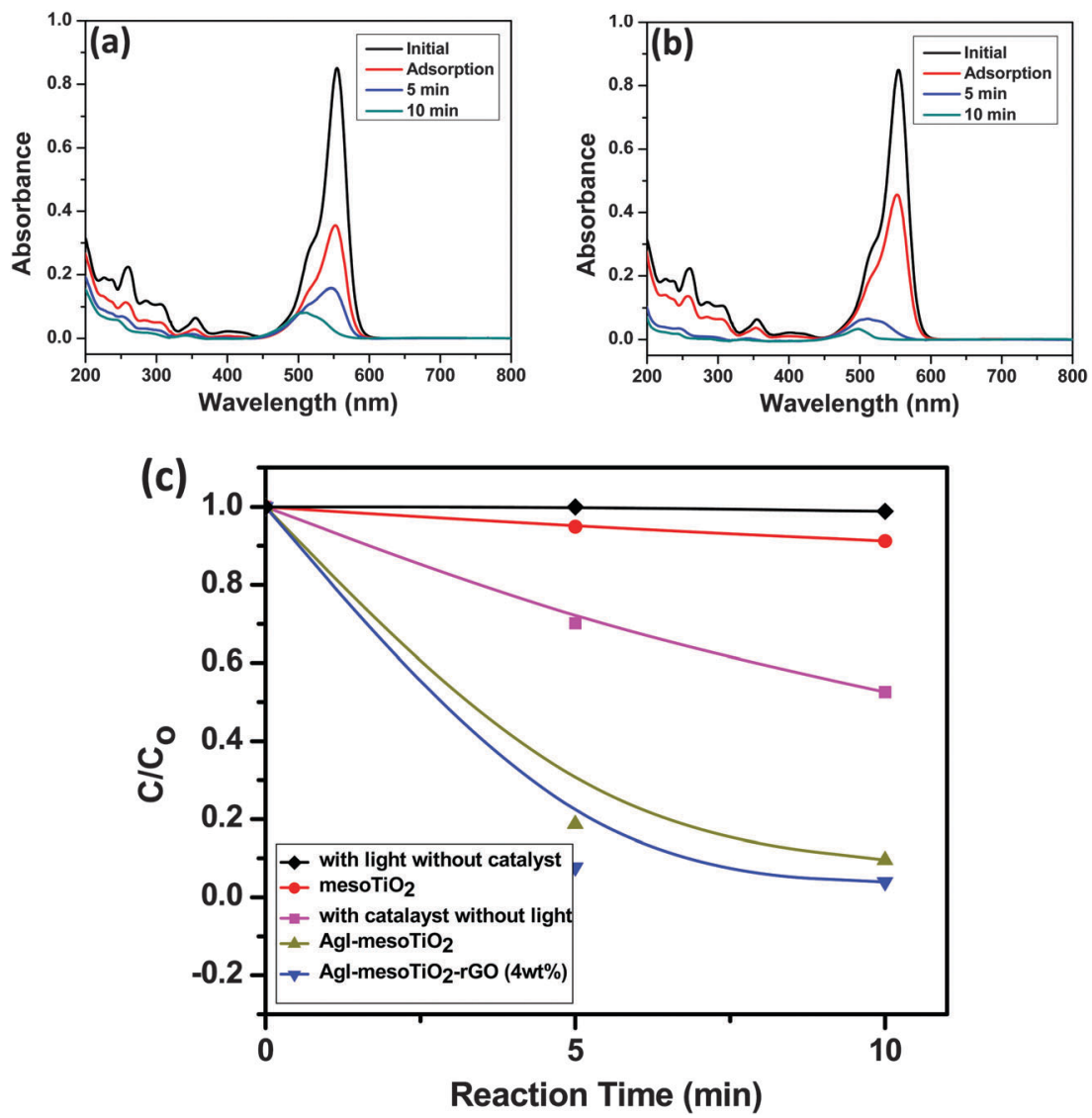

Fig. 9 Absorbance spectra of RhB after photodegradation using (a) Agl-mesoTiO ${ }_{2}$, (b) Agl-mesoTiO $2-r G O$ (4 wt\%) and (c) degradation curve of RhB using different photocatalysts under visible light irradiation.

species that are involved in $\mathrm{RhB}$ degradation, $10 \mathrm{vol} \%$ of triethanolamine (TEOA), as an effective $\mathrm{h}^{+}$scavenger was added into the $\mathrm{RhB}$ reaction solution. The rate of $\mathrm{RhB}$ degradation was drastically suppressed, i.e. only $4 \%$ of RhB degradation was noticed [Fig. 10(a)]. This result confirmed that the photoinduced holes $\left(\mathrm{h}^{+}\right)$are one of the main reactive species for the degradation of RhB. Recently, Xue et al. reported that $\mathrm{O}_{2}{ }^{-}$is the main reactive species for MO degradation using the AgI$\mathrm{TiO}_{2}$ photocatalyst. ${ }^{32}$ In order to further prove the degradation process induced by photogenerated electrons in the photocatalytic degradation of MO, another experiment was performed under a $\mathrm{N}_{2}$ atmosphere as shown in Fig. 10(b). A high purity $\mathrm{N}_{2}$ gas was continuously purged throughout the reaction process under ambient conditions, which eliminates the dissolved oxygen content from the reaction solution and thereby prevents the formation of $\mathrm{O}_{2}{ }^{-} \cdot$. As a result, only $35 \%$ in $\mathrm{MO}$ degradation was observed after $90 \mathrm{~min}$ of visible light illumination instead of $92 \%$ under normal atmospheric conditions. This reduction in the \% degradation $(\sim 60 \%)$ in the presence of $\mathrm{N}_{2}$ gas is a clear evidence that $\mathrm{O}_{2}^{-} \cdot$ radicals are the main responsible reactive species for MO degradation. On the other hand, around $10 \%$ decrease on the percentage degradation of other dye RhB under a $\mathrm{N}_{2}$ atmosphere was observed, which clearly showed that the degradation of $\mathrm{RhB}$ was not influenced by $\mathrm{O}_{2}{ }^{-}$ radicals. These results clearly emphasized that MO degradation mainly depends on superoxide radicals, whereas RhB degradation depends on photogenerated holes and ${ }^{\bullet} \mathrm{OH}$ radicals.

Similarly, the difference in the photocatalytic activity for the degradation of RhB and MO with and without rGO can be described using above observations. The possible photocatalytic reaction pathway mechanism of $\mathrm{RhB}$ and $\mathrm{MO}$ over the $\mathrm{rGO}$ supported photocatalyst is illustrated in Fig. 11. When AgImesoTiO ${ }_{2}-\mathrm{rGO}$ was irradiated with visible light, electrons from the valence band $(\mathrm{VB})$ of $\mathrm{AgI}$ are excited to the conduction band (CB) of AgI leaving positively charged holes in the VB. Since the CB energy level of $\mathrm{TiO}_{2}$ is lower than the $\mathrm{CB}$ of AgI, photogenerated electrons can easily move to the $\mathrm{CB}$ of $\mathrm{TiO}_{2}$. Moreover, interfacial carrier separations take place with the help of rGO. Since the $\mathrm{CB}$ work function of $\mathrm{TiO}_{2}$ matches with the $\mathrm{CB}$ work function of graphene, reported elsewhere, ${ }^{37}$ electrons can be easily transferred from the $\mathrm{CB}$ of $\mathrm{TiO}_{2}$ to rGO. As a result, the oxygen sites can readily accept electrons and undergo reduction reaction to generate more $\mathrm{O}_{2}{ }^{-}$radicals. Thus, the presence of rGO in photocatalysts can produce an excess amount of reactive $\mathrm{O}_{2}{ }^{-} \cdot$ radicals due to the good electron acceptor and transporter behaviour of rGO. Therefore, the ultrafast transportation of photogenerated electrons over the $\mathrm{rGO}$ sheet can directly reduce $\mathrm{O}_{2}$ to produce $\mathrm{O}_{2}{ }^{-}$radicals which leads to enhanced MO degradation (around 60\% higher). On the other hand, the degradation of $\mathrm{RhB}$ is facilitated only through the $\mathrm{h}^{+}$but not 

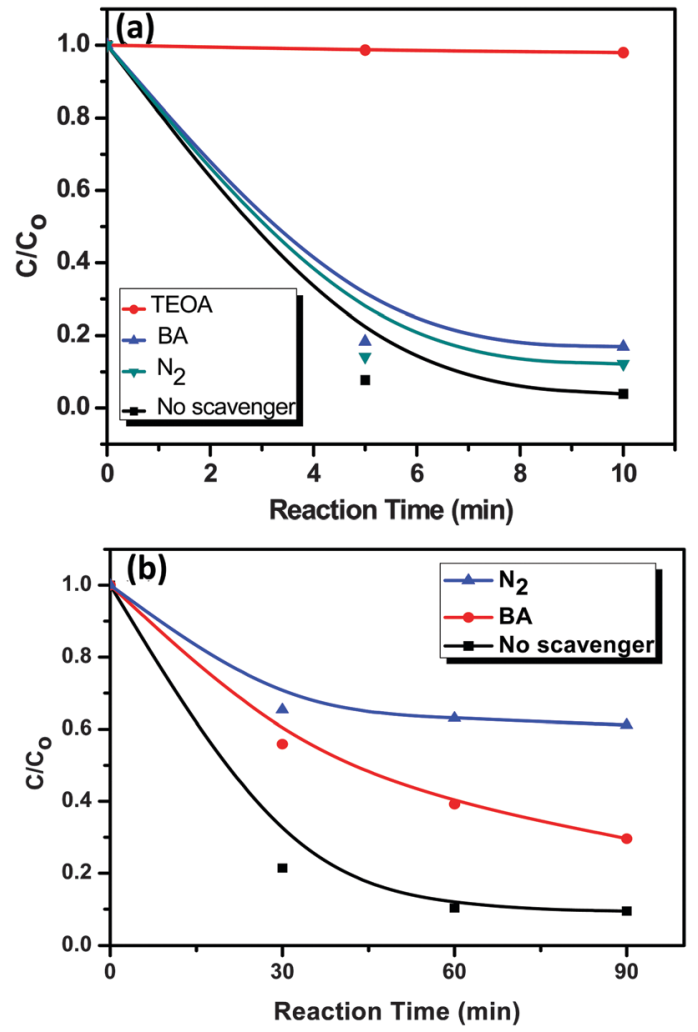

Fig. 10 A series of trapping experiments for the degradation of (a) RhB and (b) $\mathrm{MO}$ over the $\mathrm{Agl}-$ mesoTiO $2-r G O$ photocatalyst under visible light irradiation.

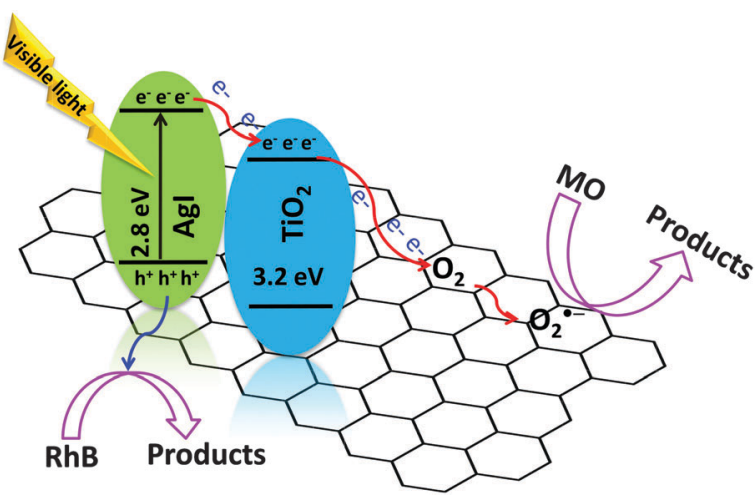

Fig. 11 Plausible photocatalytic reaction pathway mechanism of RhB and $\mathrm{MO}$ over the Agl-mesoTiO $2-r G O$ photocatalyst.

through either ${ }^{\bullet} \mathrm{OH}$ or $\mathrm{O}_{2}{ }^{-\bullet}$ radicals as discussed above. As a matter of fact, rGO is not a good carrier of $\mathrm{h}^{+}$which resulted in only a slight enhancement in RhB degradation by the addition

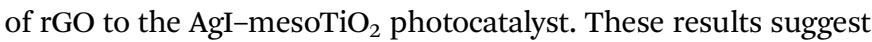
that the rate of photocatalytic degradation not only depends on rGO but also on the nature of the organics.

In order to evaluate the specific role of ultrasound in the photocatalytic degradation activity, the AgI-mesoTiO ${ }_{2}-\mathrm{rGO}$ photocatalyst was prepared in the absence of ultrasound and photodegradation was carried out using MO. It is known that the physical effects of ultrasound can influence the physical and functional properties of materials due to the shear forces generated during acoustic cavitation. ${ }^{56,57}$ As is shown in Fig. 12(a), the photocatalyst prepared in the absence of ultrasound exhibits only $60 \%$ photodegradation, whereas the photocatalyst prepared in the presence of ultrasound shows $92 \%$ of MO degradation. The preparation of photocatalysts using ultrasound might help in increasing the surface active sites of the photocatalysts apart from particle size reduction. Thus, higher active sites can increase the reactive species generation on the surface of the photocatalysts. ${ }^{58,59}$ As shown in Fig. 12(b), the XRD patterns of the rGO supported photocatalysts prepared in the presence of ultrasound shows more crystalline phases compare to that of the photocatalyst synthesized in the absence of ultrasound. This includes an added advantage for the better enhancement in photodegradation reaction. Furthermore, shear forces of ultrasound not only helps in binding the AgI nanoparticles strongly on the surface of $\mathrm{TiO}_{2}$ nanoparticles but also enhances the uniform distribution and loading of particles on rGO as clearly seen from FE-SEM images (see Fig. 3), As a result, the transport of photo-formed electrons is highly facilitated in the interfacial regions in the presence of rGO. Therefore, the present result strongly suggests that during the synthesis of nanomaterials, ultrasound plays a vital role in enhancing the photocatalytic efficiency of catalysts.

As discussed earlier, the degradation of both dyes is independent of each other, i.e., the MO dye depends on superoxide
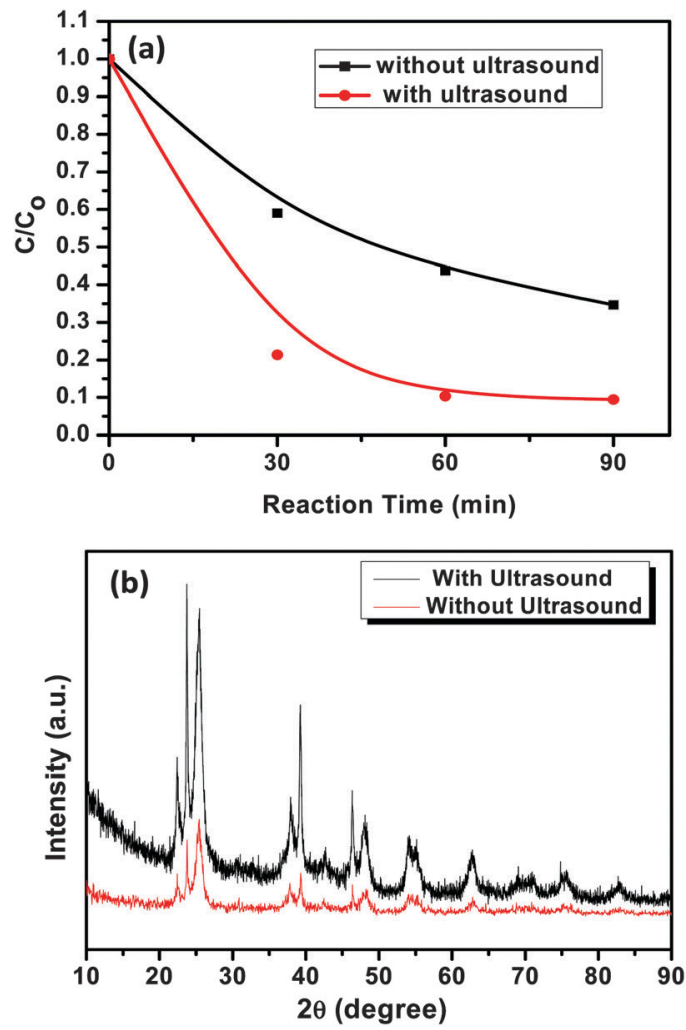

Fig. 12 (a) Photodegradation of $\mathrm{MO}$ using $\mathrm{Agl}-$ mesoTiO $_{2}-\mathrm{rGO}$ (4 wt\%) photocatalyst prepared with and without ultrasound and (b) XRD patterns of $\mathrm{Agl}-\mathrm{mesOTiO}_{2}-\mathrm{rGO}$ photocatalysts prepared with and without ultrasound. 
radicals (conduction band electrons), whereas $\mathrm{RhB}$ depends on holes that are produced in the valence band. In order to check the mechanism again, an additional experiment was carried out with a mixed dye solution which consisted of both RhB and MO. Fig. 13, shows the photodegradation of mixed dyes using the AgI-mesoTiO 2 -rGO (4 wt $\%$ ) photocatalyst. The absorption peaks of both dyes centered at 464 and $554 \mathrm{~nm}$ correspond to $\mathrm{MO}$ and $\mathrm{RhB}$, respectively. As can be seen from Fig. 13, 90\% of RhB degradation is observed within 15 min and $70 \%$ of MO photocatalytic degradation is noted in $90 \mathrm{~min}$ of visible light irradiation. The rate of degradation of both dyes is $\sim 10 \%$ less than that of individual dye degradation. This is interesting that both dyes are degraded by the prepared catalysts independently without affecting each other. Therefore, the above mixed dye degradation results strongly support the fact that photoinduced holes and superoxide radicals are the reactive species for RhB and MO degradation, as described in trapping experiments (see Fig. 10).

Transient photocurrent measurements were carried out to provide further evidence for our proposed photocatalysis mechanism of rGO loaded AgI-mesoTiO ${ }_{2}$. The transient photocurrent responses of mesoTiO $_{2}$, AgI-mesoTiO 2 and AgI-meso$\mathrm{TiO}_{2}-\mathrm{rGO}$ photocatalysts irradiated under visible light are shown in Fig. 14(a). It is seen from Fig. 14(a) that photocurrents generated for all samples are quite stable and reproducible with several on-off cycles. Notably, the AgI-mesoTiO ${ }_{2}-\mathrm{rGO}$ composite exhibits highest photocurrent response than bare mesoTiO $_{2}$ and AgI-mesoTiO ${ }_{2}$. Firstly, the AgI present in the composites can absorb visible light and generate photoinduced electron-hole pairs. The exact matching at the conduction band energy levels between $\mathrm{AgI}$ and $\mathrm{TiO}_{2}$ causes the separation of photogenerated electron-hole pairs into free electrons and holes at the $\mathrm{AgI}-\mathrm{TiO}_{2}$ interfaces. Secondly, the rGO in composites can efficiently separate and transport the carriers due to its superior electrical conductivity with excellent electron acceptor and transporter properties. The charge transfer behavior and the interfacial charge carrier separation of the photocatalysts were investigated from electrochemical impedance analysis. The Nyquist plots of

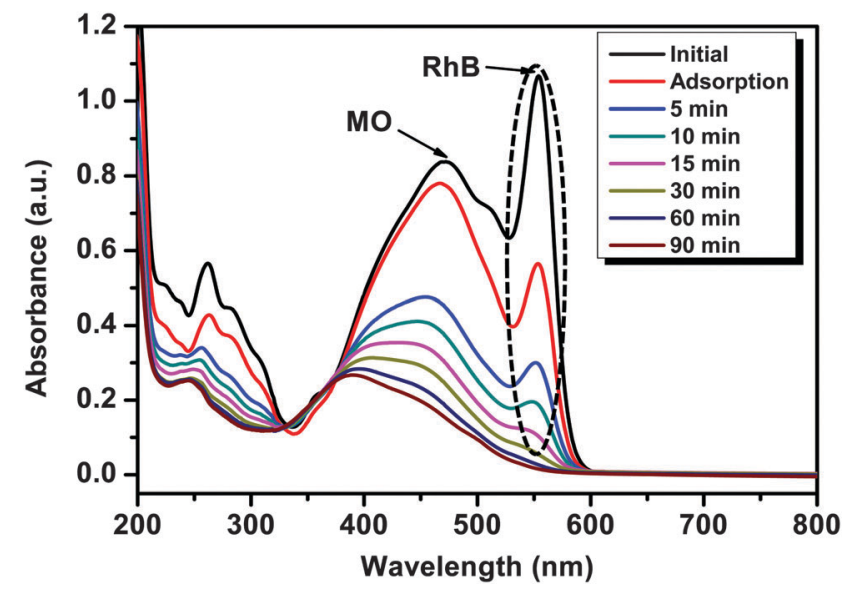

Fig. 13 Photodegradation of mixed dyes using the Agl-mesoTiO $2-r G O$ (4 wt\%) photocatalyst. (a)
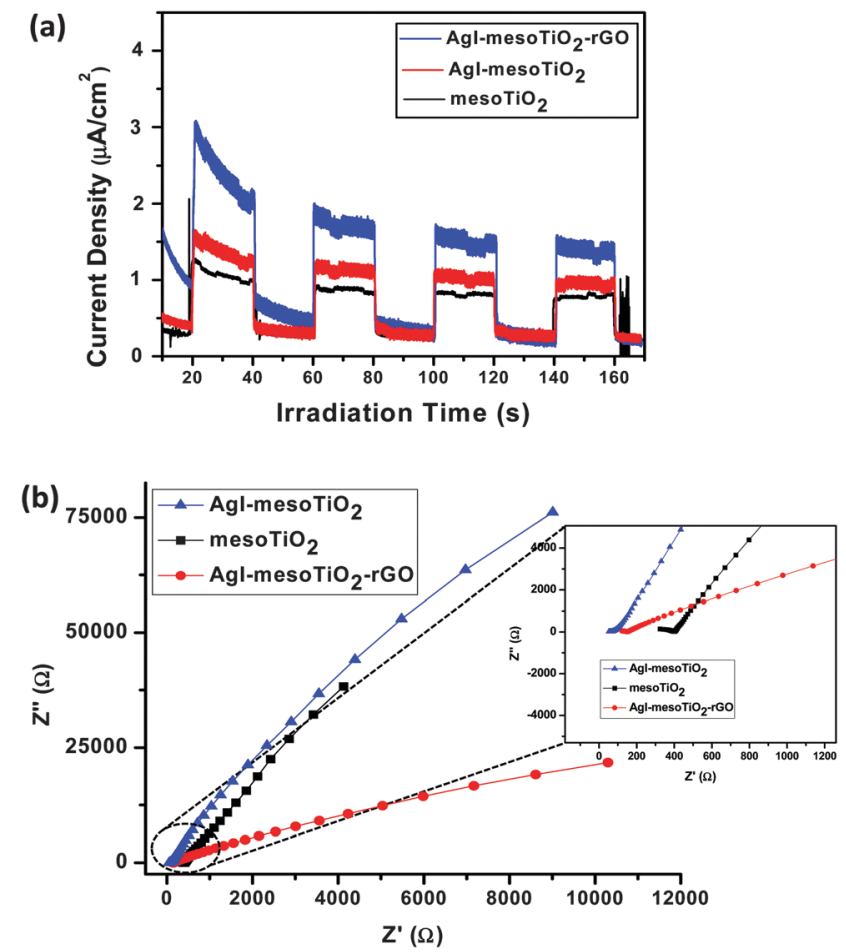

Fig. 14 (a) Transient photocurrent response of mesoTiO 2 Agl-mesoTiO and $\mathrm{Agl}-\mathrm{mesOTiO}_{2}-\mathrm{rGO}$ photocatalysts under visible light irradiation and (b) Nyquist plot of the mesoTiO $2, \mathrm{Agl}-\mathrm{mesoTiO}_{2}$ and $\mathrm{Agl}-\mathrm{mesoTiO}_{2}-\mathrm{rGO}$ photocatalysts (inset image: magnified view of the Nyquit plot).

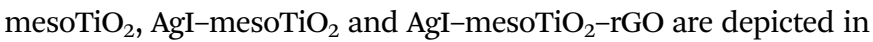
Fig. 14(b). As can be seen from the inset of Fig. 14(b), the arc radius of the $\mathrm{AgI}-\mathrm{mesoTiO}_{2}-\mathrm{rGO}$ is smaller as compared to that of bare mesoTiO $_{2}$ and AgI-mesoTiO $2-$ rGO. This observation strongly suggests that the rGO in composites can assist the efficient separation of photogenerated electron-hole pairs, which is in good agreement with transient photocurrent measurements.

\section{Conclusions}

In this study, the rGO supported AgI-mesoTiO ${ }_{2}$ photocatalyst was synthesized via a simple ultrasonic method. This composite showed extended absorbance in the visible region and subsequently reduced the bandgap compared to bare AgImesoTiO ${ }_{2}$ and mesoTiO ${ }_{2}$ photocatalysts. The $4 \mathrm{wt} \%$ rGO loaded composite exhibited high photocatalytic activity for the degradation of both MO and RhB dyes. The remarkable enhancement in the photodegradation efficiency for the degradation of MO was due to the presence of the rGO sheet, which possesses good dye adsorptivity, high carrier transportation and effective charge carrier separation properties. More interestingly, photogenerated superoxide $\left(\mathrm{O}_{2}^{-\bullet}\right)$ radicals and photo-generated holes $\left(\mathrm{h}^{+}\right)$over rGO supported photocatalysts are the main reactive species for the degradation of MO and RhB, respectively. Furthermore, the photocatalyst synthesized in the presence of ultrasound attributed much better photodegradation efficiency than the photocatalysts prepared without ultrasound. This method provides a 
simple and effective way for the development of graphene supported nanomaterials for photocatalytic applications.

\section{Acknowledgements}

This work was supported by Science and Engineering Research Board-Department of Science and Technology (SERB-DST), New Delhi, India [File No: EMR/2014/000645].

\section{References}

1 S. J. Kim and M. Shoda, Appl. Environ. Microbiol., 1999, 65, 1029-1035.

2 B. Neppolian, H. C. Choi, S. Sakthivel, B. Arabindoo and V. Murugesan, J. Hazard. Mater., 2002, 89, 303-317.

3 P. R. Gogate, M. Sivakumar and A. B. Pandit, Sep. Purif. Technol., 2004, 34, 13-24.

4 B. Neppolian, Y. Kim, M. Ashokkumar, H. Yamashita and H. Choi, J. Hazard. Mater., 2010, 182, 557-562.

5 B. Neppolian, M. V. Shankar and V. Murugesan, J. Sci. Ind. Res., 2002, 61, 224-230.

6 M. R. Hoffmann, S. T. Martin, W. Choi and D. W. Bahnemann, Chem. Rev., 1995, 95, 69-96.

7 A. Ajmal, I. Majeed, R. N. Malik, H. Idriss and M. A. Nadeem, RSC Adv., 2014, 4, 37003-37026.

8 E. Y. Kim, D. S. Kim and B. T. Ahn, Bull. Korean Chem. Soc., 2009, 30, 193-196.

9 B. Cai, J. Wang, S. Gan, D. Han, Z. Wu and L. Niu, J. Mater. Chem. A, 2014, 2, 5280-5286.

10 Y. Bi, S. Ouyang, J. Cao and J. Ye, Phys. Chem. Chem. Phys., 2011, 13, 10071-10075.

11 Y. Xu, H. Xu, J. Yan, H. Li, L. Huang, Q. Zhang, C. Huang and H. Wan, Phys. Chem. Chem. Phys., 2013, 15, 5821-5830.

12 P. Wang, B. Huang, X. Qin, X. Zhang, Y. Dai and M. H. Whangbo, Inorg. Chem., 2009, 48, 10697-10702.

13 J. Cao, B. Xu, B. Luo, H. Lin and S. Chen, Appl. Surf. Sci., 2011, 257, 7083-7089.

14 C. An, J. Wang, J. Liu, S. Wang and Q. H. Zhang, RSC Adv., 2014, 4, 2409-2413.

15 R. H. Victora, Phys. Rev. B: Condens. Matter Mater. Phys., 1997, 56, 4417-4421.

16 D. N. Bose and P. A. Govindacharyulu, Bull. Mater. Sci., 1980, 2, 221-231.

17 J. Liu, Y. Yu, Z. Liu, S. Zuo and B. Li, Int. J. Photoenergy, 2012, 2012, 1-7.

18 C. Hu, X. X. Hu, L. S. Wang, J. H. Qu and A. M. Wang, Environ. Sci. Technol., 2006, 40, 7903-7907.

19 Y. Z. Li, H. Zhang, Z. M. Guo, J. J. Han, X. J. Zhao, Q. N. Zhao and S. J. Kim, Langmuir, 2008, 24, 8351-8357.

20 M. Azimzadehirani, M. Elahifard, S. Haghighic and M. Gholami, Photochem. Photobiol. Sci., 2013, 12, 1787-1794.

21 C. An, W. Jiang, J. Wang, S. Wang, Z. Ma and Y. Li, Dalton Trans., 2013, 42, 8796-8801.
22 C. J. Miller, H. Yu and T. D. Waite, Colloids Surf., A, 2013, 435, 147-153.

23 K. I. Bolotin, K. J. Sikes, Z. Jiang, M. Klima, G. Fudenberg, J. Hone, P. Kim and H. L. Stormer, Solid State Commun., 2008, 146, 351-355.

24 R. Vinoth, S. Ganesh Babu, D. Bahnemann and B. Neppolian, Sci. Adv. Mater., 2015, 7, 1443-1449.

25 R. Kim, M. Bae, D. G. Kim, H. Cheng, B. H. Kim, D. H. Kim, M. Li, J. Wu, F. Du, H. S. Kim, S. Kim, D. Estrada, S. W. Hong, Y. Huang, E. Pop and J. A. Rogers, Nano Lett., 2011, 11, 3881-3886.

26 J. Wang, C. An, J. Liu, G. Xi, W. Jiang, S. Wang and Q. H. Zhang, J. Mater. Chem. A, 2013, 1, 4718-4727.

27 P. Wang, Y. Tang, Z. Dong, Z. Chen and T. T. Lim, J. Mater. Chem. A, 2013, 15, 2827-2832.

28 H. Xu, B. W. Zeiger and K. S. Suslick, Chem. Soc. Rev., 2013, 42, 2555-2567.

29 B. Neppolian, C. Wang and M. Ashokkumar, Ultrason. Sonochem., 2014, 21, 1948-1953.

30 W. S. Hummers, J. Richard and E. Offeman, J. Am. Chem. Soc., 1958, 80, 1339.

31 J. C. Yu, L. Zhang and J. Yu, New J. Chem., 2002, 26, 416-420. 32 B. Xue, T. Sun, J. K. Wu, F. Mao and W. Yang, Ultrason. Sonochem., 2015, 22, 1-6.

33 Z. L. Wang, D. Xu, Y. Huang, Z. Wu, L. M. Wang and X. B. Zhang, Chem. Commun., 2012, 48, 976-978.

34 S. Vadivel, M. Vanitha, A. Muthukrishnaraj and N. Balasubramanian, J. Water Process Eng., 2014, 1, 17-26.

35 Q. Huang, S. Tian, D. Zeng, X. Wang, W. Song, Y. Li, W. Xiao and C. Xie, ACS Catal., 2013, 7, 1477-1485.

36 H. Zhang, X. Lv, Y. Li, Y. Wang and J. Li, ACS Nano, 2010, 4, 380-386.

37 S. D. Perera, R. G. Mariano, K. Vu, N. Nour, O. Seitz, Y. Chabal and K. J. Balkus, ACS Catal., 2012, 2, 949-956.

38 Y. Zhang, Z. R. Tang, X. Fu and Y. Jun Xu, ACS Nano, 2010, 4, 7303-7314.

39 Z. D. Meng, L. Zhu, T. Ghosh, C. Y. Park, K. Ullah, V. Nikam and W. C. N. Oh, Bull. Korean Chem. Soc., 2012, 33, 3761-3766.

40 X. Lin, J. Xing, W. Wang, Z. Shan, F. Xu and F. Huang, J. Phys. Chem. C, 2007, 111, 18288-18293.

41 L. Chen, D. Jiang, T. He, Z. Wu and M. Chen, CrystEngComm, 2013, 15, 7556-7563.

42 K. Vinodgopal, B. Neppolian, I. V. Lightcap, F. Grieser, M. Ashokkumar and P. V. Kamat, J. Phys. Chem. Lett., 2010, 1, 1987-1993.

43 D. A. Reddy, J. Choi, S. Lee, R. Ma and T. K. Kim, RSC Adv., 2015, 5, 67394-67404.

44 D. A. Reddy, R. Ma and T. K. Kim, Ceram. Int., 2015, 41, 6999-7009.

45 B. Qiu, Y. Zhou, Y. Ma, X. Yang, W. Sheng, M. Xing and J. Zhang, Sci. Rep., 2015, 5, 8591-8596.

46 L. R. Radovic, Chemistry and Physics of Carbon, CRC Press, Taylor and Francis group, 2013.

47 S. Ganesh Babu, R. Vinoth, B. Neppolian, D. D. Dionysiou and M. Ashokkumar, J. Hazard. Mater., 2015, 291, 83-92. 
48 B. Neppolian, V. Sáez, J. G. García, F. Grieser, R. Gómez and M. Ashokkumar, J. Solid State Electrochem., 2014, 18, 3163-3171.

49 G. An, W. Ma, Z. Sun, Z. Liu, B. Han, S. Miao, Z. Miao and K. Ding, Carbon, 2007, 45, 1795-1801.

50 X. Wang, Q. Xiang, B. Liu, L. Wang, T. Luo, D. Chen and G. Shen, Sci. Rep., 2013, 3, 2007-2014.

51 K. Vignesh, A. Suganthi, B. K. Min, M. Rajarajan and M. Kang, RSC Adv., 2015, 5, 576-585.

52 Y. Z. Li, H. Zhang, Z. M. Guo, J. J. Han, X. J. Zhao, Q. N. Zhao and S. J. Kim, Langmuir, 2008, 24, 8351-8357.

53 B. Neppolian, Q. Wang, H. Yamashita and H. Choi, Appl. Catal., A, 2007, 333, 264-271.
54 B. Neppolian, A. Bruno, C. L. Bianchi and M. Ashokkumar, Ultrason. Sonochem., 2012, 19, 9-15.

55 G. Yang, Z. Jiang, H. Shi, T. Xiao and Z. Yan, J. Mater. Chem., 2010, 20, 5301-5309.

56 B. Neppolian, Q. Wang, H. Jung and H. Choi, Ultrason. Sonochem., 2008, 15, 649-658.

57 S. H. Jhung, K. C. Yoo, Y. K. Hwang and J. S. Chang, Bull. Korean Chem. Soc., 2007, 28, 2401-2404.

58 M. Lirong, S. Jianjun, Z. Ming and H. Jie, Bull. Korean Chem. Soc., 2014, 35, 3521-3525.

59 S. Pankaj and M. Ashokkumar, Theoretical and Experimental Sonochemistry Involving Inorganic Systems, Springer Science \& Business Media, 2011. 